\title{
Propriétés de cohérence spatiale d'un faisceau d'harmoniques
}

\author{
L. Le Déroff, B. Carré, P. Salières, D. Joyeux* et D. Phalippou*
}

CEA-DSM/DRECAM/SPAM, CE de Saclay, bâtiment 522, 91191 Gif-sur-Yvette cedex, France

*Institut d'Optique, URA 14, BP. 147, 91403 Orsay cedex, France

\begin{abstract}
Résumé : Nous avons étudié les propriétés de cohérence spatiale d'un faisceau harmonique à 55nm dans deux expériences distinces. D'une part, nous caractérisons la taille et la divergence du faisceau au foyer d'un miroir sphérique. D'autre part, nous mesurons le degré de cohérence spatiale dans le faisceau, par une technique d'interférométrie à division de front d'onde. Les deux mesures caractérisent une bonne cohérence spatiale du faisceau, paramètre $\mathrm{M}^{2} \approx 2$ et degré de cohérence spatiale $\gamma \approx 0.5$, qui peut être utilisée dans les applications.
\end{abstract}

\section{QUALITE DU FAISCEAU HARMONIQUE}

Nous avons caractérisé un faisceau de rayonnement harmonique à $55 \mathrm{~nm}$, focalisé avec un miroir multicouche molibdène-silicium sphérique $(\mathrm{f} \#=40)$. La distribution spatiale de l'éclairement a été caractérisée dans la région du foyer par la méthode du foucaultage, en fonction de la pression et de la longueur du milieu générateur[1].

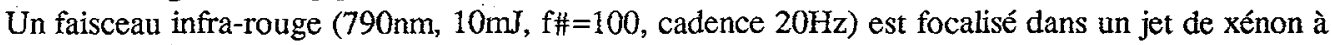
une intensité de $10^{13}-10^{14} \mathrm{~W} / \mathrm{cm}^{2}$. La buse du jet est de profil rectangulaire $(300 \mu \mathrm{m} \times 3 \mathrm{~mm})$, une rotation du jet permet de changer la longueur du milieu générateur. Derrière le multicouche et le filtre en aluminium utilisé (épaisseur $100 \mathrm{~nm})$, le spectre est dominé par les harmoniques $13(61 \mathrm{~nm})$ et $15(53 \mathrm{~nm})$.

En déplaçant une lame dans le faisceau par pas de $2 \mu \mathrm{m}$, on effectue des coupes horizontales (x), et verticales $(y)$, en différentes positions $-10 \mathrm{~mm}<z<10 \mathrm{~mm}$ de part et d'autre du meilleur foyer. L'analyse des profils détermine la taille horizontale du faisceau $\Delta x(z)$ (verticale $\Delta y$ ) à $70 \%$ de l'énergie totale transmise (faisceau gaussien $\Delta x(z)=1.04 w_{x}(z)$, où $w_{x}(z)$ est la demi-largeur du faisceau à $1 / \mathrm{e}^{2}$ ). Les profils $\Delta x(z)$ sont analysés avec les paramètres usuels $\mathrm{w}_{\mathrm{x} 0}$, la taille au foyer, $\mathrm{M}_{\mathrm{x}}{ }^{2}$, le nombre de fois que le faisceau est limité par la diffraction. Dans l'analyse, ces deux paramètres sont liés à la divergence totale derrière le miroir : $\operatorname{div}_{\mathrm{tot}}=\left(2 \mathrm{M}_{\mathrm{x}}^{2} \lambda\right) /\left(\pi \mathrm{W}_{\mathrm{x} 0}\right)$. Les paramètres $\mathrm{W}_{\mathrm{x} 0}, \mathrm{M}_{\mathrm{x}}^{2}$ ainsi que div $\mathrm{tot}_{\mathrm{tot}}$ sont mesurés en fonction de la pression amont (fig. 1a, $1 \mathrm{~b}$ et $1 \mathrm{c}$ ), la pression utile est typiquement 10 fois plus faible que la pression amont. La taille $w_{x 0}$ reste voisine de $10 \mu \mathrm{m}$ pour les deux types de jet.

Pour un jet long ( $3 \mathrm{~mm}$, symbole plein) la divergence totale après focalisation augmente sensiblement avec la pression (fig. 1c). Cette variation se traduit par une augmentation de $M_{\mathrm{x}}{ }^{2}$ de 3 à 6 . Dans le cas d'un jet court ( $300 \mu \mathrm{m}$, symbole ouvert) la divergence est plus faible et quasi constante. La valeur du paramètre $\mathrm{M}_{\mathrm{x}}{ }^{2}$ est en conséquence plus faible, entre 2 et 4 . L'augmentation de la divergence et du facteur $\mathrm{M}^{2}$ avec la densité et la longueur du jet s'explique par la défocalisation du faisceau IR dans le milieu partiellement ionisé. Dans les conditions de meilleure focalisation avec le miroir sphérique $(\mathrm{f} \#=40$ ), on obtient au foyer un éclairement harmonique de l'ordre de $10^{11} \mathrm{~W} / \mathrm{cm}^{2}$.

\section{MESURE DE LA COHERENCE SPATIALE}

Nous avons utilisé un interféromètre du type bimiroir de Fresnel pour caractériser la cohérence spatiale d'un faisceau harmonique $\left(\lambda_{13}=61 \mathrm{~nm}\right.$ dominant, $\left.\lambda_{15}=53 \mathrm{~nm}\right)$. La géométrie fixe la distance $\mathrm{d}$ entre les rayons du faisceau incident qui interfèrent à une distance $\mathrm{Z}$ derrière l'interféromètre $\left(\mathrm{d} \approx 10^{-3} \mathrm{Z}\right)$. On mesure ainsi la cohérence spatiale à l'échelle de 1 à $3 \mathrm{~mm}$, dans un faisceau de diamètre $5 \mathrm{~mm}$, dont le profil est typiquement gaussien.

A la distance $Z$ derrière l'interféromètre, les deux moitiés du faisceau se superposent et interferent, On peut modéliser la distribution d'éclairement $I_{\mathrm{lot}}(\mathrm{x})$ sur le détecteur par la figure d'interférence de 2 
faisceaux, d'enveloppes radiales demi-gaussiennes, $I_{\mathrm{g} 1}(\mathrm{x})$ et $\mathrm{I}_{\mathrm{g} 2}(\mathrm{x})$, présentant un degré de cohérence spatiale $\gamma$ :

$$
I_{t o t}(x)=I_{g 1}(x)+I_{g 2}(x)+2 \gamma \sqrt{I_{g 1}(x) I_{g 2}(x)} \operatorname{coskx}
$$

où $\mathrm{k}$ représente la fréquence spatiale des franges. La figure $2 \mathrm{a}$ montre un profil horizontal $\mathrm{I}_{\mathrm{tor}}(\mathrm{x}) \mathrm{de}$ la figure d'interférence, superposition des deux interférogrammes pour $\lambda_{H 13}$ et $\lambda_{H 15}$. On détermine tout d'abord les paramètres de $I_{g 1}(x), I_{g 2}(x)$ en filtrant les modulations rapides du profil $I_{t o t}(x)$; l'analyse du profil filtré (fig. 2b) inclut la diffraction, clairement visible, par l'arête du bimiroir. L'amplitude du pic à la fréquence $\mathrm{k}$ de la transformée de Fourier du signal réel permet alors de calculer le degré de cohérence $\gamma(\mathrm{d})$. La validité de la méthode est vérifiée par comparaison du profil réel et du profil reconstruit à partir de l'analyse (fig. 2c).

On a mesuré des degrés de cohérence relativement élevés $\gamma>0.5$ jusqu'à des distances $\mathrm{d} \leq 3 \mathrm{~mm}$. La cohérence spatiale du faisceau harmonique dépend également des paramètres de la génération d'harmoniques tels que la pression dans le jet, la distance jet-foyer et l'éclairement laser [2,3].

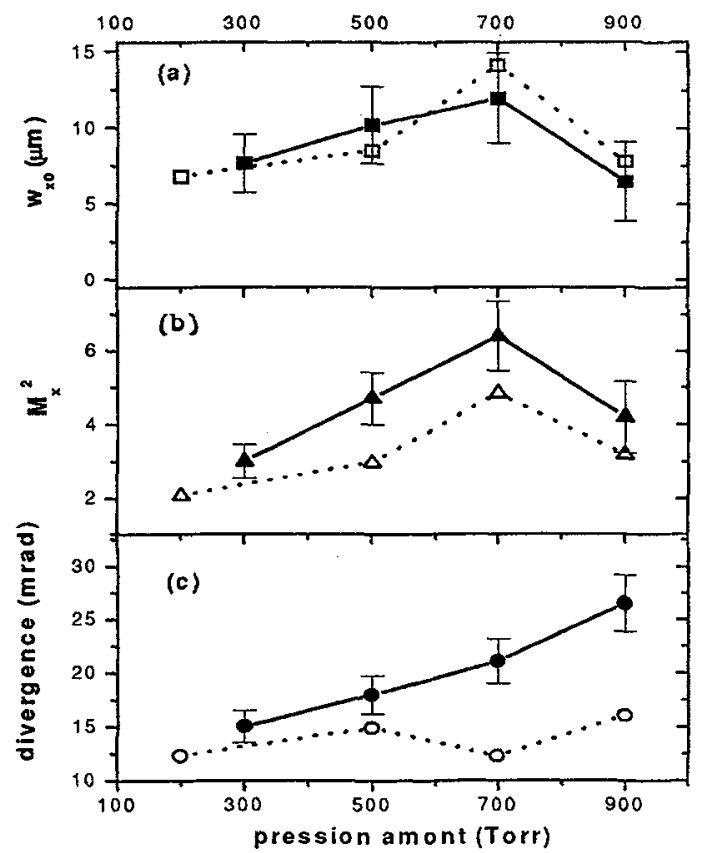

Figure 1: Variation de (a) $w_{x 0}$, (b) $M_{x}^{2}$ et (c) divergence horizontale en fonction de la pression amont pour un jet court (symbole ouvert) et un jet long (symbole plein).
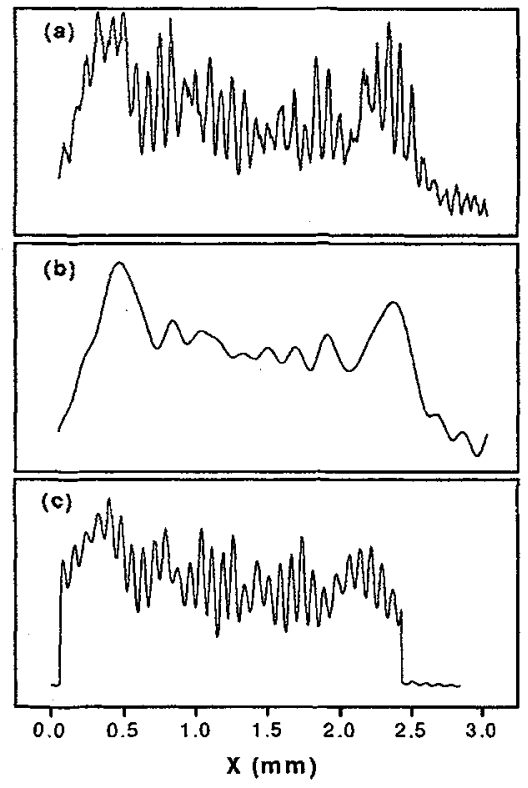

Figure 2: Profil d'interférences (a) mesuré, (b) lissé et (c) reconstruit, pour une distance $\mathrm{d}=1.8 \mathrm{~mm}$.

\section{Références}

1. L. Le Déroff, P. Salières, B. Carré, Opt. Lett. 23, 1544 (1998).

2. T. Ditmire, E. T. Grumbell, R. A. Smith, J. W. G. Tisch, D. D. Meyerhofer, M. H. R. Hutchinson, Phys. Rev. Lett. 77, 4756 (1996).

3. En cours de publication. 